Revue internationale P.M.E.

Économie et gestion de la petite et moyenne entreprise

\title{
Incertitude environnementale, aversion au risque du dirigeant et investissement productif des petites et moyennes entreprises
}

\section{Jacques Bernard Sauner-Leroy}

Volume 12, numéro 3, 1999

URI : https://id.erudit.org/iderudit/1008658ar

DOI : https://doi.org/10.7202/1008658ar

Aller au sommaire du numéro

Éditeur(s)

Presses de l’Université du Québec

ISSN

0776-5436 (imprimé)

1918-9699 (numérique)

Découvrir la revue

Citer cet article

Sauner-Leroy, J. B. (1999). Incertitude environnementale, aversion au risque du dirigeant et investissement productif des petites et moyennes entreprises. Revue internationale P.M.E., 12(3), 33-57. https://doi.org/10.7202/1008658ar
Résumé de l'article

Cet article a pour objet de tester empiriquement les liens entre le niveau d'investissement productif des PMI et le degré d'incertitude de leur environnement et d'aversion au risque de leur dirigeant.

Cette analyse est effectuée à partir de données issues de l'enquête qualitative de la Banque de France menée en 1996 sur les comportements stratégiques de 1439 PMI de l'industrie manufacturière française.

Quatre styles de management, correspondant à des niveaux d'incertitude et d'aversion au risque opposés, sont mis en évidence et associés à des taux d'investissement significativement différents. Le recours à une régression multiple permet ensuite de confirmer et d'affiner les résultats obtenus.

Il en ressort notamment que les phénomènes combinés d'incertitude et d'aversion au risque apparaissent comme des déterminants pertinents de la décision d'investir. Les conclusions de cette étude indiquent que, nonobstant un nécessaire renforcement de leur structure financière, la stimulation de l'investissement des PMI passe par des actions de sensibilisation à l'importance du recueil et du traitement de l'information stratégique ainsi que par des incitations à l'accroissement de leurs efforts en matière de dépenses en recherche et développement. 


\section{Incertitude environnementale, aversion au risque du dirigeant et investissement productif des petites et moyennes entreprises}

Jacques Bernard SAUNER-LEROY

Banque de France

MOTS CLÉS

Investissement - Irréversibilité - Dirigeant - Incertitude Aversion au risque

\section{RÉSUMÉ}

Cet article a pour objet de tester empiriquement les liens entre le niveau d'investissement productif des PMI et le degré d'incertitude de leur environnement et d'aversion au risque de leur dirigeant.

Cette analyse est effectuée à partir de données issues de l'enquête qualitative de la Banque de France menée en 1996 sur les comportements stratégiques de 1439 PMI de l'industrie manufacturière française.

Quatre styles de management, correspondant à des niveaux d'incertitude et d'aversion au risque opposés, sont mis en évidence et associés à des taux d'investissement significativement différents. Le recours à une régression multiple permet ensuite de confirmer et d'affiner les résultats obtenus.

\section{L'AUTEUR}

JaCQUES BeRnARD SAUNER-LeRor est diplômé de l'Institut d'études politiques de Paris. Il est adjoint de direction et responsable de la gestion et de l'exploitation de la base de données qualitatives de la Banque de France sur les comportements économiques et financiers des PME de l'industrie manufacturière française. Adresse: Banque de France, Direction des Entreprises, 45-1367, 31, rue Croix-des-Petits-Champs, 75049 Paris Cedex 01. Tel. 330142923648. 
Il en ressort notamment que les phénomènes combinés d'incertitude et d'aversion au risque apparaissent comme des déterminants pertinents de la décision d'investir. Les conclusions de cette étude indiquent que, nonobstant un nécessaire renforcement de leur structure financière, la stimulation de l'investissement des PMI passe par des actions de sensibilisation à l'importance du recueil et du traitement de l'information stratégique ainsi que par des incitations à l'accroissement de leurs efforts en matière de dépenses en recherche et développement.

\section{ABSTRACT}

The purpose of this study is to examine the links between the level of investment of small and medium-sized firms and the degree of uncertainty of business environnement and the degree of risk aversion of business leaders.

The analysis makes use of qualitative data collected by the Banque de France in a national survey conducted in 1996 on the strategic behavior of 1436 small and medium-sized French industrial firms.

Four management styles associated with significant differences in business uncertainty, risk aversion and investment rates are distinguished. Then a multiple regression model confirms and completes the results.

It is demonstrated that the combination of uncertainty of business environment and risk aversion is negatively correlated with investment rate. In order to spur small and medium-sized firms to increase their productive investments the findings suggest to encourage business leaders to collect as much strategic informations as possible and to raise their $R \& D$ spendings.

\section{RESUMEN}

El objeto del presente artículo consiste en probar empíricamente los vínculos existentes entre el nivel de inversión productiva de las PyMEs y los grados de incertidumbre con respecto a su entorno y de aversión al riesgo de sus dirigentes.

Este análisis se desarrolló a partir de los datos procedentes de la encuesta cualitativa que el Banque de France realizó en 1996 sobre los comportamientos estratégicos de 1439 PyMEs de la industria manufacturera francesa.

Se ponen de manifiesto cuatro estilos de gerencia correspondientes a niveles de incertidumbre y de aversión al riesgo opuestos, y que se encuentran asociados a tasas de inversión significativamente diferentes. A continuación, se recurre a una regresión múltiple que permite confirmar y afinar los resultados obtenidos.

De lo anterior se desprende principalmente que la combinación de los fenómenos de incertidumbre y de aversión al riesgo figuran como determinantes pertinentes de la decisión de invertir. Las conclusiones de este estudio indican que, a pesar de que sea necesario un fortalecimiento de su estructura financiera, el fomento de la inversión de las pequeñas y medianas empresas requiere acciones orientadas a despertar la conciencia con respecto a la importancia de la reunión y el tratamiento de la información estratégica, así como incentivos para el incremento de sus esfuerzos en materia de gastos de investigación y desarrollo. 


\section{ZUSAMMENFASSUNG}

Dieser Artikel hat zum Ziel, empirisch den Zusammenhang zwischen dem produktiven Investitionsniveau der kleinen und mittleren Industrieunternehmen und den Risikofaktoren ihrer Umwelt und der mangelnden Risikobereitschaft der Führungskräfte zu testen.

Die Analyse stützt sich auf die Resultate einer qualitativen Umfrage im Jahre 1996 der Banque de France“ über die strategischen Zielsetzungen von 1439 kleinen und mittleren Unternehmen der französischen Fertigungsindustrie.

Vier Führungsstile, geprägt von Unsicherheit und mangelnder Risikobereitschaft wurden in Verbindung mit erheblich unterschiedlichen Investitionsentscheidungen gebracht. Der Rückgang der Investitionsbereitschaft bestätigt und verfeinert die erhaltenen Resultate.

Das Phänomen zeigt, dass Unsicherheit kombiniert mit mangelnder Risikobereitschaft die Investitionsentscheide massgebend beeinflussen. Die Studie kommt zum Schluss, dass ungeachtet einer nötigen Verstärkung der Kapitalstruktur die Anregung zu Investitionen bei kleinen und mittleren Industrieunternehmen durch Beschaffung und Verwaltung von strategischen Informationen sowie durch Anreize zur Vergrösserung der Ausgaben im Bereich Forschung und Entwicklung, gefördert werden kann.

L'analyse de la décision d'investir des entreprises est traditionnellement abordée en référence à des concepts d'ordre financier, à travers notamment des notions telles que la valeur actuelle nette ou le délai de récupération des capitaux engagés. Or, ramener la décision d'investir à un simple calcul d'actualisation, dans lequel la détermination du taux d'intérêt occupe une place centrale, ou de comparaison entre un décaissement immédiat et des recettes futures peut apparaître par trop réducteur d'une réalité sans doute beaucoup plus complexe. En effet, alors que l'environnement des entreprises devient de plus en plus mouvant et compétitif, altérant ainsi les prévisions d'évolution d'activité et de rentabilité, le fort degré d'inertie que comporte un projet d'investissement productif, lié au fait qu'il est par nature difficilement réversible, en constitue une caractéristique majeure dont il paraît difficile de faire abstraction.

Depuis les travaux de Henry (1974), les développements de la littérature consacrée à la théorie de l'investissement ont mis l'accent sur l'impact restrictif que pouvaient avoir les phénomènes d'irréversibilité et d'incertitude. Toutefois, si l'influence de ces effets est désormais parfaitement admise sur un plan strictement microéconomique, sa formalisation dans le cadre de modèles économétriques s'est heurtée jusqu'à présent à des difficultés pratiques de mesure de l'incertitude, d'une part, et, d'autre part, à une forte hétérogénéité des comportements individuels, notamment sur les sources d'incertitude, rendant quasi inopérante leur agrégation sur le plan macroéconomique (Galiegue, 1996 ; Bourdieu, Coeure et Sedillot, 1997). 
Par ailleurs, la nature des objectifs poursuivis par les chefs d'entreprise - qui, par référence à la typologie établie par Julien et Marchesnay (1987), peut schématiquement se décliner entre une recherche de sécurité ou de pérennité (entrepreneur «PIC ») et une volonté de croissance et d'acceptation du risque qui en découle (entrepreneur « $\mathrm{CAP} »)$ - ne saurait également rester sans conséquence sur leurs comportements en matière d'investissement, notamment en ce qui concerne les petites et moyennes entreprises (Girard, 1997). Or ce dernier aspect, l'impact de l'aversion au risque du dirigeant sur la politique d'investissement de la firme, a été très peu analysé, et encore moins testé empiriquement. L'une des principales raisons que l'on peut évoquer pour expliquer cet état de fait, ainsi que les problèmes de mesure de l'incertitude relevés précédemment, concerne l'absence de données «empiriques » permettant de cerner individuellement chacun de ces deux paramètres.

Partant de ce constat, l'objectif de cette étude est d'intégrer dans l'analyse de la décision d'investir une mesure objective de l'incertitude et de l'aversion au risque du dirigeant ainsi que de combiner ces deux dimensions afin d'enrichir l'approche de ce thème. Pour ce faire, nous nous sommes appuyé sur l'exploitation de données qualitatives relatives à 1439 petites et moyennes entreprises de l'industrie manufacturière française.

En outre, pour surmonter l'obstacle lié à l'agrégation de comportements individuels hétérogènes, il nous est apparu intéressant de construire une typologie de styles de management associés à des niveaux différenciés d'incertitude et d'aversion au risque.

La première partie présente une synthèse des principaux développements théoriques récents relatifs à la décision d'investissement. La deuxième partie aborde la méthodologie qui a été retenue et la troisième, les principaux résultats et conclusions qui se dégagent. Il en ressort qu'au-delà des constats déjà bien établis concernant les contraintes de débouchés, de financement ou d'appartenance sectorielle, les phénomènes d'incertitude et d'aversion au risque apparaissent effectivement comme des déterminants pertinents et complémentaires dans la prise de décision d'investir du dirigeant.

\section{Approches théoriques}

L'analyse de la littérature récente consacrée à la décision d'investir fait ressortir, au-delà des critères traditionnels tels que le coût du capital et le profit anticipé, les deux thèmes majeurs que sont l'incertitude et l'aversion au risque, auxquels sont associées un certain nombre de dimensions complémentaires. 


\subsection{L'incertitude et l'aversion au risque : deux dimensions structurantes de la décision d'investir}

\section{L'incertitude, source d'attentisme à l'égard de la décision d'investir}

L'étude de la décision d'investissement de l'entreprise est un domaine qui a déjà fait l'objet de nombreux travaux depuis ceux entrepris par Keynes et Marshall. Deux grands courants se sont développés autour des modèles en $Q$ (Tobin, 1969) - rapport entre la valeur boursière des actifs et le coût de remplacement actuel de ceux-ciet de ceux de type accélérateur / coûts relatifs / profits issus des travaux de Jorgensen (1963). Les modèles issus de ces travaux, très schématiquement, se sont longtemps contentés de considérer le processus d'investissement comme un ajustement instantané du stock de capital existant au stock de capital désiré défini par la recherche de la maximisation du profit courant. Ce faisant, ces travaux théoriques se sont heurtés à la difficulté de tenir compte de la véritable nature de l'investissement, à savoir le pari sur l'avenir qu'il représente en raison notamment de son très fort degré d'inertie. Les phénomènes liés à l'irréversibilité de l'investissement et à l'incertitude sur les états futurs de la nature ont ainsi longtemps été ignorés par les modèles classiques, non parce qu'ils étaient inconnus mais parce qu'ils étaient considérés comme impropres à toute formalisation théorique ou trop dépendants de l'imperfection des marchés.

Si Arrow (1968) s'est penché sur le problème de la considération de l'irréversibilité en matière de prise de décision d'investir, il ne tenait pas compte des effets de l'incertitude. En revanche, d'autres travaux les intégraient (Mills, 1959, 1962; Hartman, 1972) mais dans le cadre classique des modèles à choix réversibles.

L'irréversibilité de l'investissement en environnement incertain a été considérée pour la première fois par Henry (1974), puis s'est développée, à partir du début des années 1980, avec les travaux de Bernanke (1983) et surtout ceux de Mac Donald et Siegel $(1985,1986)$ qui intègrent l'apport de la théorie financière, et plus particulièrement celle des options, à l'analyse de la décision d'investir. Les travaux de Dixit (1989, 1991, 1992) et de Pyndyck (1988, 1991), enfin, ont abouti à la définition d'un modèle conceptuel bien formalisé.

Dans ce cadre, l'irréversibilité se définit par rapport à la réalisation d'un projet dont, d'une part, le coût est irrécupérable et asymétrique (le coût du désinvestissement est supérieur à la simple perte du montant de l'investissement initial $)^{1}$ et qui, d'autre part, grève les occasions d'investissement dans le futur.

1. La transformation d'un capital acquis sur le marché en un capital spécifique, propre à l'usage de chaque entreprise donne à l'investissement un caractère irréversible dans la mesure où il est plus coûteux de faire puis de défaire que de ne rien faire (Bourdieu, Coeure et Sedillot, 1997). 
De fait, lorsque le niveau de l'information dont disposent les agents économiques sur leur environnement s'accroît avec le temps (dans la mesure où chaque période de temps qui s'écoule apporte de l'information supplémentaire sur l'état de l'environnement), le moment de la prise de décision ne leur est pas indifférent parce que l'attente permet de réduire l'incertitude relative au futur, c'est-à-dire le risque de perturbations futures de l'environnement, et autorise donc des choix plus pertinents que ceux qui seraient pris dans l'immédiat. Ainsi, plus le projet est contraignant pour l'avenir (irréversibilité) et plus ce dernier est incertain (incertitude), plus les décisions seront empreintes de « conservatisme» (attente) [Henry, 1974].

L'introduction de «l'effet d'irréversibilité » aboutit ainsi à renverser l'analyse traditionnelle qui prévalait depuis le modèle de Hartman (1972) et selon laquelle l'incertitude exerçait un effet positif sur le niveau de l'investissement, même en présence de coûts d'ajustement (Abel, 1983) : il est admis désormais que l'incertitude sur les états futurs de la nature conduit, à l'inverse, à restreindre l'investissement (Caballero, 1991 ; Abel et Eberly, 1994).

Dans ces conditions, le critère « traditionnel » d'évaluation d'un projet d'investissement qui consiste, sur le plan microéconomique, à comparer le coût actuel du projet à la somme actualisée des flux de revenus futurs anticipés, et dans lequel le taux d'intérêt occupe une place essentielle, perd une partie de sa pertinence. Ainsi, en environnement incertain, la question ne se pose plus de savoir s'il est économiquement rentable d'investir mais s'il est opportun de le faire aujourd' hui plutôt que demain, sachant que l'attente préserve la possibilité d'entreprendre éventuellement dans de meilleures conditions et qu'elle est susceptible de réduire l'incertitude sur l'avenir.

\section{L'aversion au risque du dirigeant renforce les effets de l'incertitude sur la décision d'investir}

Si la littérature économique consacrée au phénomène d'investissement s'est penchée ces dernières années sur les effets de l'incertitude environnementale sur la décision d'investir, à notre connaissance, très peu de travaux ont traité de l'aversion au risque de l'entrepreneur en environnement incertain et de ses conséquences sur sa décision d'investir. Or, «la présence d'aversion au risque, rarement prise en compte dans les modèles, devrait renforcer l'effet négatif de l'irréversibilité sur la décision d'investissement» (Bourdieu, Coeure et Sedillot, 1997).

Des travaux analysant les liens entre l'aversion au risque, l'incertitude et la prise de décision stratégique ont cependant déjà été menés. Julien et Marchesnay (1987) ont notamment établi une typologie du comportement des entrepreneurs en fonction du degré de risque qu'ils acceptent d'assumer pour atteindre les objectifs prioritaires qu'ils se sont fixés. Dans ce cadre, les auteurs distinguent deux catégories d'entrepreneurs : les «PIC » (pérennité, indépendance, croissance), dont l'une des principales préoccupations est d'assurer la survie de leur firme, ce qui en fait 
des agents qui ont une grande aversion au risque, et les « CAP » (croissance, autonomie, pérennité), pour lesquels la valorisation de leur patrimoine, principal objectif poursuivi, passe par une prise de risque supérieure. Cette typologie a notamment été reprise et complétée par Silvestre et Goujet (1996) qui distinguent quatre types de comportements stratégiques de dirigeants selon les caractéristiques de l'environnement :

TABleau 1

Typologie de styles managériaux selon le degré de prédictabilité de l'environnement

\begin{tabular}{lcc}
\hline & Environnement repérable & Environnement non repérable \\
\hline Dirigeant «CAP» & comportement proactif & comportement opportuniste \\
\hline Dirigeant «PIC» & comportement réactif & comportement attentiste \\
\hline
\end{tabular}

Ainsi, selon les auteurs, le comportement « proactif» sera adopté par un dirigeant qui, dans un environnement lisible, investit en prévision des évolutions de ce dernier, alors que le comportement réactif, dans le même type d'environnement, sera observé chez un dirigeant qui investit après avoir constaté ces mêmes évolutions.

Le dirigeant «opportuniste » est celui qui profite, dans un environnement difficilement prévisible, de toute possibilité d'action en fonction des occasions qui s'offrent à lui, alors que «l'attentiste » préfère différer sa prise de décision afin de bénéficier des informations que son attente lui permettra d'obtenir sur l'environnement et limiter ainsi ses risques.

L'analyse de la littérature propose une typologie de styles managériaux attachés à la prise de décision d'investir. C'est cette typologie qui sera reprise et testée dans cette étude.

\subsection{La prise en considération de dimensions complémentaires}

En plus des deux dimensions structurantes que constituent les phénomènes d'incertitude et d'aversion au risque, on relève également dans la littérature un certain nombre de facteurs complémentaires susceptibles d'influer sur la décision d'investir.

\section{Le degré de participation du dirigeant au capital de la firme}

Jensen et Meckling (1976) ont notamment mis en évidence des comportements différenciés à l'égard du risque selon que le dirigeant est lui-même propriétaire de l'entreprise qu'il dirige ou simple mandant, agissant pour le compte de ses actionnaires. Ainsi, selon cette théorie dite de «l'agence», «lorsque la part du capital possédée par le manager décroît, sa motivation à consacrer un effort significatif à 
des activités créatives telles que la recherche de nouvelles occasions d'investissement rentable diminue ». Cette aversion pour le risque est susceptible d'apparaître en raison de l'existence de «relations d'agence » qui peuvent émerger lorsque les dirigeants de l'entreprise sont mandatés par les propriétaires. Dans ce type de relations, les managers et les actionnaires peuvent poursuivre des objectifs antinomiques : les premiers auront tendance à privilégier des actions visant notamment à assurer leur pérennité dans leurs fonctions ou à limiter les «coûts personnels et les anxiétés » qui pourraient résulter de l'acquisition de nouvelles technologies associées aux investissements, ce qui les conduira à adopter un comportement minimisant la prise de risque, alors que les seconds, surtout s'ils disposent d'un portefeuille d'actifs diversifié, seront plus favorables à un degré de risque supérieur puisque celui-ci est susceptible de maximiser les profits de l'entreprise et donc leur richesse.

\section{La taille}

Les risques et l'incertitude liés à la décision d'investir peuvent toutefois être sensiblement limités par l'accès à l'information et le suivi des marchés (études marketing ou commerciales, par exemple) qui permettent de mieux appréhender les perspectives d'évolution de la demande et de l'offre, la stratégie des concurrents, le risque d'apparition de produits de substitution ou de nouvelles technologies de produit ou de production (voir notamment Betbeze, 1990).

Or, il existe dans ce domaine une très forte asymétrie entre les grandes entreprises, qui peuvent mobiliser d'importants moyens humains ou financiers pour acquérir l'information nécessaire, et les PME, dont les ressources sont plus limitées et qui sont donc plus exposées au risque et à l'incertitude (voir notamment Richet, 1994). Dans ces conditions, il peut paraitre opportun de surseoir à la décision d'investir et espérer que les décisions des entreprises du même secteur apporteront des informations sur la rentabilité de l'investissement (Chamley et Gale, 1994).

Par ailleurs, le risque client tend désormais à être de plus en plus assumé par les PME. Depuis la fin des années 1980, en effet, les grandes entreprises ont eu tendance à réduire leurs engagements en tant que prêteurs du crédit interentreprises et à transférer cette charge aux petites et moyennes entreprises, qui sont amenées à y consacrer une partie de leurs fonds propres (Dietsch et Kremp, 1997). En outre, les PME sont plus susceptibles de se trouver en situation de dépendance à l'égard de leurs clients (mêmes auteurs). Ces éléments peuvent être à l'origine du fait qu'elles hésitent davantage à lancer des programmes d'investissement productif.

\section{La structure concurrentielle du marché}

La structure concurrentielle, mesurée par la taille du marché et le degré de banalisation des actifs, peut aussi avoir des effets négatifs sur l'investissement. En effet, 
si le marché servi par l'entreprise est trop étroit, les coûts fixes de développement et de lancement de nouveaux produits ne pourront être amortis assez rapidement, ce qui peut engendrer des comportements de mise en sommeil de projets d'investissement en attendant que le marché se développe (Bourdieu, Coeure et Sedillot, 1997). Le même type de comportement peut apparaitre si les technologies maîtrisées par les différents concurrents sont relativement banalisées, car l'imitation risquerait d'annuler assez rapidement les surplus de gains espérés par l'investissement résultant d'une innovation de produit ou de procédé (mêmes auteurs).

\section{La contrainte de financement}

La décision d'investir reste toutefois indissociable de la question du choix du mode de financement. À cet égard, Myers et Majluf (1984) ont mis en évidence une hiérarchie dans les préférences des dirigeants : l'autofinancement serait privilégié au recours à l'endettement, lequel est lui-même préféré à l'augmentation de capital. Cette hiérarchie dans les modes de financement, dont la pertinence pour l'analyse de la finance des PME est désormais bien admise dans la littérature (voir notamment à ce sujet les travaux de Calof, 1985 ou ceux de Holmes et Kent, 1991), amène à considérer le niveau d'autofinancement de la firme comme une variable essentielle dans l'analyse du processus de décision d'investir. Il en va de même de la capacité à faire appel à l'emprunt intermédié, les travaux de Belletante $(1991,1995)$ sur le territoire financier ayant notamment mis en exergue l'importance du recours à l'endettement bancaire comme source de capitaux pour les PME, contrairement aux financements désintermédiés (émissions d'obligations ou d'actions) qui leur restent assez largement inaccessibles.

\section{Méthodologie}

\subsection{Les étapes de l'analyse}

L'analyse se décompose en 2 étapes.

Dans la mesure où il ressort de la revue de littérature que l'incertitude et l'aversion au risque représentent des dimensions structurantes de la prise de décision d'investir, les interrelations entre ces deux dimensions et la décision d'investissement sont en premier lieu analysées par le biais d'une typologie de groupes d'entrepreneurs inspirée des travaux de Silvestre et Goujet (1996).

Dans une deuxième étape, la prise en considération de facteurs complémentaires permet d'affiner l'analyse et de caractériser plus précisément chaque style managérial. Une analyse de régression permet ensuite de confirmer les résultats qui se dégagent. 


\subsection{Les bases de données}

Les informations économiques sont extraites de la base de données qualitatives de la Banque de France (SESAME) sur les PMI de l'industrie manufacturière dont les effectifs sont compris entre 35 et 2000 salariés (annexe I).

L'étude porte sur les entreprises appartenant aux secteurs de l'industrie agroalimentaire, des biens de consommation, de l'industrie automobile et de l'industrie textile qui ont été enquêtées en 1996, dont les effectifs n'excèdent pas 500 salariés et qui, au cours de la période considérée, n'ont pas fait l'objet de rachat par une autre entreprise et n'ont, par ailleurs, pas effectué d'opérations de croissance externe. L'échantillon de départ se compose de 1896 entreprises.

Les données financières sont issues du Fichier bancaire des entreprises de la Banque de France (FIBEN). Les valeurs sont calculées en moyenne sur trois ans (1993-1995), afin de «lisser» leur évolution sur moyenne période et de dégager ainsi une dimension structurelle. Les valeurs extrêmes ou aberrantes pour chaque indicateur ont été éliminées.

\subsection{La mesure de l'effort d'investissement productif}

L'effort d'investissement productif est mesuré par le taux d'investissement productif moyen des entreprises entre 1993 et 1995. Le taux d'investissement se définit comme le rapport exprimé en pourcentage entre l'investissement en immobilisations corporelles d'exploitation et la valeur ajoutée. Afin de limiter l'influence des valeurs extrêmes, les entreprises se situant dans le dernier centile de la distribution de cette variable ont été exclues de l'analyse (ce qui correspond à 15 entreprises). En raison de l'existence de valeurs manquantes pour ce ratio, l'échantillon étudié ne comprend plus que 1439 firmes.

\subsection{Les variables qualitatives utilisées pour la construction de la typologie des styles managériaux associés à la décision d'investir}

\subsubsection{Variables représentatives de l'incertitude liée à l'évolution de l'environnement}

Le questionnaire n'ayant pas été élaboré spécifiquement pour cette étude ( $c f$. annexe I), il ne comprend pas de variables mesurant directement l'incertitude du chef d'entreprise quant à l'évolution de l'environnement. Cette dimension est examinée à travers des questions relatives à ses prévisions d'évolution de son marché principal et de sa vision de l'évolution du métier de son entreprise. La mesure de 
l'incertitude retenue ici est donc inversement liée au degré de prévisibilité de l'environnement de la firme. Il s'agit d'une incertitude environnementale perçue par le dirigeant.

\section{TABLEAU 2}

Description des variables mesurant l'incertitude environnementale

\begin{tabular}{lcc}
\hline Variable & Question posée & Échelle de réponse \\
\hline $\begin{array}{l}\text { Prévision d'évolution } \\
\text { du marché principal }\end{array}$ & $\begin{array}{c}\text { "Sur combien de mois estimez- } \\
\text { vous réalistes les prévisions } \\
\text { d'évolution de votre marché } \\
\text { principal ?» }\end{array}$ & Nombre en mois \\
\hline $\begin{array}{l}\text { Vision de l'évolution } \\
\text { du métier }\end{array}$ & $\begin{array}{c}\text { "La direction a une vision de } \\
\text { l'évolution du métier } \\
\text { de l'entreprise sur : }\end{array}$ & $\begin{array}{c}\text { De 1 an (ou moins) à 5 ans } \\
\text { (ou plus) }\end{array}$ \\
\hline
\end{tabular}

Pour les besoins de l'analyse, ces deux variables ont été transformées en variables binaires. Le seuil de deux ans (sur une échelle allant jusqu'à 5 et plus) a été retenu comme valeur « critique » pour la variable « évolution du métier » et celui de 12 mois pour celle ayant trait à l'évolution du marché. Sont ainsi considérées comme évoluant dans un environnement peu lisible les PMI pour lesquelles la prévision d'évolution du métier est inférieure ou égale à deux ans et les prévisions d'évolution du marché inférieures ou égales à 12 mois. Pour les autres, l'environnement est considéré comme lisible, donc faiblement empreint d'incertitude.

\subsubsection{Variables représentatives du degré d'aversion au risque du dirigeant}

Le risque est communément mesuré soit par la volatilité d'une variable sur une période donnée, soit par un indicateur synthétique de vulnérabilité financière. Aucune de ces deux approches n'a été retenue, car ce que nous cherchons à mesurer c'est l'acceptation par le chef d'entreprise d'une probabilité de perte, totale ou partielle, en capital associée à une décision d'investissement. En d'autres termes, il s'agit d'un risque de détérioration de la situation financière ou de la capacité bénéficiaire de l'entreprise lié à l'incertitude qui pèse sur le retour sur investissement. Dans ce cadre, prendre des risques revient, pour un chef d'entreprise, à incorporer cette probabilité de perte dans sa prise de décision d'investir en contrepartie d'une espérance de gain élevée.

Le degré d'aversion au risque a été mesuré par deux variables déduites de la typologie établie par Julien et Marchesnay (1987) entre les entrepreneurs «PIC » (pérennité, indépendance, croissance) et les entrepreneurs «CAP » (croissance, autonomie, pérennité). 
Ces deux variables sont représentatives des objectifs poursuivis par les chefs d'entreprise et des manœuvres qui leur sont associées. Ainsi, dans la mesure où les entrepreneurs «CAP» recherchent en priorité la valorisation de leur capital en exploitant les opportunités de croissance, le degré d'acceptation du risque (et donc a contrario celui d'aversion au risque) peut être estimé par référence à la plus ou moins grande propension du dirigeant à poursuivre des objectifs de gains de parts de marché en liaison avec des actions visant à assurer le développement de sa firme (deux types d'actions, recensées lors de l'enquête, ont été retenues à cet effet : augmentation de la production et augmentation de l'effort commercial).

\section{TABLEAU 3}

Description des variables mesurant le degré d'acceptation du risque du dirigeant

\begin{tabular}{|c|c|c|}
\hline Dimension & Mesure & Échelle de mesure \\
\hline $\begin{array}{l}\text { Objectif de croissance } \\
\text { de la firme }\end{array}$ & $\begin{array}{l}\text { Objectif d'accroissement } \\
\text { de la part de marché à court, } \\
\text { moyen et long terme }\end{array}$ & $\begin{array}{c}\text { De } 0 \text { (absence) } \\
\text { à } 3 \text { (recherche prioritaire) }\end{array}$ \\
\hline $\begin{array}{l}\text { Manœuvres orientées vers } \\
\text { le développement de la firme }\end{array}$ & $\begin{array}{c}\text { Augmentation de la } \\
\text { production ou de l'effort } \\
\text { commercial }\end{array}$ & $\begin{array}{c}\operatorname{De} 0(\text { absence }) \\
\text { à } 4 \text { (orientation prioritaire) }\end{array}$ \\
\hline
\end{tabular}

Pour les besoins de l'analyse, un score, produit des deux variables, a été calculé. Ce score (comprenant sept valeurs entre zéro et 12) a ensuite été transformé en variable binaire de sorte que l'on puisse distinguer les entreprises pour lesquelles la recherche de la croissance de la part de marché n'est pas prioritaire. La valeur 1, correspondant à une orientation faiblement marquée par l'acceptation du risque telle qu'elle a été définie précédemment, a été retenue comme seuil «critique». Sont ainsi considérées comme adverses au risque les firmes dont le score est inférieur ou égal à un.

\subsubsection{Construction d'une typologie de comportements en matière d'investissement}

Le croisement des deux dimensions binaires « incertitude » et « aversion au risque » permet d'identifier quatre groupes d'entreprises définis selon leur comportement stratégique. Ces groupes correspondent à ceux qui avaient été définis a priori : comportement «proactif » (environnement lisible, acceptation du risque), « réactif » (environnement lisible, aversion au risque), « opportuniste » (environnement peu lisible, acceptation du risque), «attentiste » (environnement peu lisible, aversion au risque). 


\section{TABLEAU 4}

Répartition des entreprises par style de management du dirigeant

\begin{tabular}{lcc}
\hline & $\begin{array}{c}\text { Environnement } \\
\text { peu lisible }\end{array}$ & $\begin{array}{c}\text { Environnement } \\
\text { lisible }\end{array}$ \\
\hline Faible aversion au risque & $\begin{array}{c}\text { Opportuniste } \\
\text { (149 entreprises })\end{array}$ & $\begin{array}{c}\text { Proactif } \\
\text { (419 entreprises) }\end{array}$ \\
\hline Forte aversion au risque & $\begin{array}{c}\text { Attentiste } \\
\text { (248 entreprises) }\end{array}$ & $\begin{array}{c}\text { Réactif } \\
\text { (584 entreprises) }\end{array}$ \\
\hline
\end{tabular}

\subsection{Variables qualitatives représentatives des facteurs complémentaires}

\subsubsection{Variables mesurant le niveau d'information du chef d'entreprise}

Ce concept est analysé à travers un «score » calculé en fonction des réponses fournies à la question se rapportant aux types d'informations utilisées par le chef d'entreprise dans la formulation de sa stratégie (plus le score est élevé, plus le niveau d'informations utilisées l'est aussi). Ce score est étalonné entre un et cinq.

\subsubsection{Variables représentatives du degré de contrôle de l'entreprise par le dirigeant}

La variable utilisée permet de classer les entreprises en fonction du degré de contrôle du capital par les dirigeants : absence de participation, participation simple, influence notable, contrôle conjoint, contrôle exclusif. Ces modalités sont intégrées sur une échelle comprise entre un (absence de participation) à cinq (contrôle exclusif).

\subsubsection{Variables mesurant la taille de l'entreprise}

La taille de l'entreprise est mesurée par les effectifs moyens au cours de la période étudiée. La définition de la PMI qui a été retenue est celle de l'INSEE (moins de 500 salariés, avec toutefois un minimum de 35 salariés correspondant au seuil à partir duquel l'enquête SESAME est réalisée).

\subsubsection{Variables mesurant la dimension du marché principal}

La dimension du marché principal est mesurée par une question se rapportant à la dimension géographique de ce marché (quatre choix sont proposés : dimension régionale, nationale, européenne, internationale). Ces modalités sont notées de un (dimension régionale) à quatre (dimension internationale). 


\subsubsection{Variables mesurant le degré de banalisation des technologies maîtrisées par l'entreprise}

Cette dimension est évaluée à travers une variable a contrario mesurant le degré de différenciation des technologies de production de l'entreprise (échelle de un [faible] à cinq [fort]).

\subsubsection{La prise en considération de contraintes internes ou externes à l'entreprise permet d'affiner l'analyse}

Les contraintes externes qui peuvent peser sur la décision d'investir concernent essentiellement la contrainte de débouchés, mesurée par le taux de croissance moyen du marché principal de l'entreprise au cours des deux dernières années. Il s'agit d'une variable issue de l'enquête.

Les contraintes internes à l'entreprise sont essentiellement d' ordre financier.

La contrainte financière est ainsi mesurée par deux indicateurs comptables : le taux d'autofinancement (capacité d'autofinancement / valeur ajoutée) et le poids des charges financières (intérêts / excédent brut d'exploitation). Ce dernier ratio étant intégré en tant qu'indicateur de la capacité d'endettement de la firme auprès du système bancaire (plus le ratio est élevé, moins la firme peut s'endetter).

\section{Résultats et discussion}

\section{Comparaison des styles de management}

Le tableau 5 présente les résultats des tests statistiques (analyse de variance, tests non paramétriques) effectués entre les quatre types de comportement stratégique sur la variable mesurant le taux d'investissement productif. Ce tableau présente la moyenne de cet indicateur pour chaque type de comportement, et révèle l'existence de différences de moyenne significatives au seuil de $5 \%$ entre ces groupes.

Le groupe des entrepreneurs « proactifs » apparaît comme celui dont le comportement d'investissement est le plus dynamique, à l'inverse de celui des firmes dont le dirigeant est plutôt « attentiste ». Les « réactifs » et les « opportunistes » apparaissent, quant à eux, dans une position intermédiaire par rapport aux deux autres groupes. Ces résultats tendent ainsi à confirmer l'hypothèse selon laquelle la conjugaison d'une forte aversion au risque et d'une faible prévisibilité de l'environnement est défavorable à la décision d'investir, et inversement. 


\section{TABLEAU 5}

\section{Comparaison des styles de management en fonction du taux d'investissement}

\begin{tabular}{lcc}
\hline $\begin{array}{l}\text { Taux d'investissement } \\
\text { productif }(\boldsymbol{\%})\end{array}$ & Incertitude élevée & Incertitude faible \\
\hline Faible aversion au risque & Opportuniste & \\
& $9,05 \%$ & Proactif \\
& $(=)$ & $11,94 \%$ \\
& Attentiste & $(+++)$ \\
\hline Forte aversion au risque & $7,35 \%$ & Réactif \\
& $(-)$ & $9,55 \%$ \\
& & $(=)$ \\
\hline$(+++)$ ou $(-)$ & signifie que la moyenne est significativement supérieure ou inférieure à celles des trois \\
& autres groupes. &
\end{tabular}

Afin de mieux caractériser chacun des quatre types de comportement de chef d'entreprise, un recoupement a été opéré avec les facteurs de contingence testés au préalable. Le tableau 6 en présente la synthèse :

\section{TABLEAU 6}

\section{Relations entre les facteurs complémentaires et les styles de management}

\begin{tabular}{lcccc}
\hline & Attentistes & Opportunistes & Réactifs & Proactifs \\
\hline Information stratégique & - & - & + & + \\
\hline Effectifs & $103(-)$ & $97(=)$ & $125(+++)$ & $106(=)$ \\
\hline Croissance du marché & $-2 \%(-)$ & $-0,95 \%(=)$ & $+0,23 \%(+)$ & $+0,46 \%(+)$ \\
\hline $\begin{array}{l}\text { Différenciation des } \\
\text { technologies de production }\end{array}$ & - & $=$ & + & + \\
\hline $\begin{array}{l}\text { Poids des intérêts } \\
\text { Taux d'autofinancement }\end{array}$ & $10,55 \%(-)$ & $13,63 \%(=)$ & $14,05 \%(=)$ & $17,28 \%(+++)$ \\
\hline $\begin{array}{l}\text { Degré de contrôle de } \\
\text { l'entreprise par le dirigeant }\end{array}$ & $3,83(+)$ & $3,77(=)$ & $3,48(-)$ & $3,59(=)$ \\
\hline $\begin{array}{l}\text { Dimension géographique } \\
\text { du marché principal }\end{array}$ & $2,71(+)$ & $2,57(=)$ & $2,6(=)$ & $2,51(-)$ \\
\hline
\end{tabular}

(+) indique que la moyenne est significativement supérieure à celle indiquée (-) au seuil de $5 \%$.

$(+++)$ ou $(-)$ indique que la moyenne est significativement supérieure ou inférieure au seuil de $5 \%$ à celles des trois autres groupes.

(=) indique que la moyenne ne s'oppose pas de manière significative à celles des autres groupes, sauf celles indiquées (-) ou (+++). 
Ces résultats confirment l'importance du facteur débouché et du taux d'autofinancement. Ils tendent en outre à indiquer une relation inverse entre la dimension du marché et le taux d'investissement. Ce résultat surprenant provient en fait d'un effet «secteur » induit par l'appartenance ou la non-appartenance à l'industrie agroalimentaire. Les PMI enquêtées recensées dans ce secteur se caractérisent en effet à la fois par des niveaux de taux d'investissement très supérieurs et une dimension géographique de leur marché principal sensiblement inférieure aux autres PMI enquêtées. C'est cet effet qui ressort dans l'opposition entre «attentistes » et " proactifs » sur le facteur « dimension géographique du marché », les premiers étant sous-représentés et les seconds, surreprésentés au sein de l'industrie agroalimentaire.

Par ailleurs, le degré de contrôle de l'entreprise par le dirigeant ou la taille ne semblent pas apporter d'informations supplémentaires.

L'analyse des facteurs «structurants » et « complémentaires » de la décision d'investir permet d'établir le double constat suivant :

- l'aversion au risque apparaît d'autant plus élevée (attentistes et réactifs) que les contraintes financières sont fortes;

- l'incertitude est d'autant plus faible (proactifs et réactifs) que le degré d'utilisation de l'information stratégique est élevé.

\section{Analyse de régression}

Le tableau 7 présente les régressions effectuées sur le taux d'investissement productif avec et sans prise en considération de l'appartenance sectorielle (régressions 1 et 2). Pour les besoins de l'analyse, les données quantitatives (taux d'investissement productif, taux d'autofinancement) ont été transformées de façon à «normaliser » leurs distributions, tout en veillant à ce que ces nouvelles variables aient un coefficient de corrélation très élevé avec la variable d'origine. Les autres données, issues de l'enquête, sont introduites en tant que variables binaires (annexe II). La dimension géographique du marché principal, pour les motifs évoqués précédemment, et le poids des intérêts dans l'excédent brut d'exploitation, en raison de sa forte corrélation avec le taux d'autofinancement, ont été exclus de l'analyse.

L'analyse des régressions fait ressortir les éléments suivants :

- la confirmation de l'importance du facteur de l'appartenance sectorielle dans l'explication du taux d'investissement, notamment, que les secteurs de l'agroalimentaire et du textile sont particulièrement dynamiques, à l'inverse de celui de l'habillement et des fourrures.

- l'opposition entre les groupes « proactifs » et « attentistes » semble pertinente pour expliquer des différences de comportements à l'égard de la décision d'investir ; cela confirme le fait que la combinaison de l'aversion au risque et de l'incertitude affecte négativement l'investissement des PMI; 


\section{TABLEAU 7}

Régression linéaire sur le taux d'investissement productif

\begin{tabular}{|c|c|c|c|c|}
\hline & (1) & Min & $\operatorname{Max}$ & (2) \\
\hline $\mathbf{N}$ & 1371 & & & 1371 \\
\hline Opportunistes & $0,207(0,175)$ & 0 & 1 & $0,204(0,183)$ \\
\hline Proactifs & $0,468 * *(0,139)$ & 0 & 1 & $0,4649 * *(0,144)$ \\
\hline Réactifs & $0,180(0,130)$ & 0 & 1 & $0,210(0,135)$ \\
\hline $\begin{array}{l}\text { Niveau d'information } \\
\text { intermédiaire }\end{array}$ & $0,07(0,109)$ & 0 & 1 & $0,102(0,113)$ \\
\hline Niveau d'information élevé & $0,299 * *(0,116)$ & 0 & 1 & $0,336 * *(0,122)$ \\
\hline Autofinancement & $0,256 * * *(0,021)$ & 1 & 11 & $0,322 * * *(0,022)$ \\
\hline Différenciation technologique & $0,098^{* *}(0,035)$ & 1 & 5 & $0,115^{* *}(0,037)$ \\
\hline Dirigeant mandaté & $0,315 * *(0,115)$ & 0 & 1 & $0,449 * * *(0,119)$ \\
\hline Participation simple & $-0,247(0,200)$ & 0 & 1 & $-016(0,209)$ \\
\hline Influence notable & $0,255(0,205)$ & 0 & 1 & $0,333(0,215)$ \\
\hline Contrôle conjoint & $0,009(0,15)$ & 0 & 1 & $0,089(0,158)$ \\
\hline Effectifs 250 à 499 & $0,526 * *(0,176)$ & 0 & 1 & $0,271 *(0,127)$ \\
\hline Effectifs 100 à 249 & $0,35 * *(0,123)$ & 0 & 1 & $0,434 *(0,181)$ \\
\hline Effectifs 50 à 99 & $0,203(0,115)$ & 0 & 1 & $0,188(0,12)$ \\
\hline Croissance nulle & $0,083(0,116)$ & 0 & 1 & $0,251 *(0,12)$ \\
\hline Croissance positive & $0,372 * * *(0,111)$ & 0 & 1 & $0,578^{* * *}(0,111)$ \\
\hline NAF_15 & $1,799^{* * *}(0,165)$ & 0 & 1 & \\
\hline NAF_17 & $1,44^{* * *}(0,177)$ & 0 & 1 & \\
\hline NAF_19 & $0,646 * *(0,243)$ & 0 & 1 & \\
\hline NAF_22 & $0,92 * * *(0,186)$ & 0 & 1 & \\
\hline NAF_24 & $0,979 * * *(0,257)$ & 0 & 1 & \\
\hline NAF_29 & $0,931(0,498)$ & 0 & 1 & \\
\hline NAF_32 & $1,805(0,969)$ & 0 & 1 & \\
\hline NAF_33 & $0,681(0,388)$ & 0 & 1 & \\
\hline NAF_34 & $0,74 * *(0,228)$ & 0 & 1 & \\
\hline NAF_36 & $0,755^{* * *}(0,179)$ & & & \\
\hline Constante & $2,335^{* * *}(0,209)$ & & & $2,833 * * *(0,2024)$ \\
\hline $\mathrm{R}^{2}$ & 0,314 & & & 0,236 \\
\hline $\operatorname{Adj} . R^{2}$ & 0,30 & & & 0,227 \\
\hline $\mathrm{F}$ & $23,671 * * *$ & & & $26,133^{* * *}$ \\
\hline
\end{tabular}

$* \operatorname{sign}<0,05, * * \operatorname{sign}<0,01, * * * \operatorname{sign}<0,001$

Note: Les chiffres entre parenthèses représentent l'écart type. Les intitulés des codes NAF figurent en annexe II.

- le degré de contrôle du capital de l'entreprise par le dirigeant semble corrélé négativement avec le taux d'investissement. Plus précisément, l'opposition entre le dirigeant mandaté et le dirigeant-propriétaire apparaît comme la plus saillante, le dirigeant-propriétaire de PMI apparaissant 
moins apte à investir ; ces résultats semblent ainsi a priori infirmer, au regard de l'analyse des PMI, les hypothèses issues de la théorie de l'agence puisque cette dernière préconiserait une relation inverse. Ces résultats rejoignent en outre les constats déjà faits par des auteurs comme Chandler (1976), Landes (1957) ou Alcroft (1966) dont les travaux concluent à un comportement de type malthusien lié à la réticence à réaliser des investissements importants nécessitant une ouverture du capital et par là une perte totale ou partielle de contrôle de la firme, dans les firmes anglaises et françaises à structure familiale au XIX ${ }^{\mathrm{e}}$ siècle. La structure familiale du capital des PME / PMI constituerait donc un frein à une politique dynamique d'investissement, pour des raisons de conservation du pouvoir ou d'autonomie de décision au sein de l'entreprise. On peut aussi supposer que, dans la mesure où les parts détenues dans le capital de la PMI constituent l'essentiel de l'actif patrimonial du dirigeant-propriétaire, son aversion à l'égard du risque est plus élevée que celle d'un actionnaire disposant d'un portefeuille d'actifs diversifié.

- les variables relatives à l'information et à la différenciation de l'outil de production apparaissent également pertinentes dans le modèle, en complément de variables plus traditionnelles comme le taux de croissance du marché, la taille, au-delà du seuil des 100 salariés, ou le taux d'autofinancement.

\section{Conclusion}

Cette étude a permis de mettre en évidence le fait que, au-delà des seuls critères financiers, les phénomènes combinés d'incertitude et d'aversion au risque permettent de mieux cerner et de mieux comprendre les déterminants de la décision d'investir du dirigeant d'entreprise, mais également que d'autres dimensions qualitatives, telles que la détention du capital de l'entreprise, le degré d'informations stratégiques exploitées ou la différenciation de l'outil de production, apparaissent tout aussi pertinentes dans l'approche de ce thème.

Même si les résultats obtenus ne concernent qu'une partie des PMI de l'industrie manufacturière française, ce qui en relativise la portée, il est possible d'en tirer quelques enseignements à destination notamment des mesures publiques d'incitation à l'effort d'investissement des PMI :

- s'efforcer de réduire l'incertitude qui entoure les prises de décisions stratégiques des chefs d'entreprise en menant des actions de sensibilisation à l'importance du recueil et du traitement de l'information;

- contribuer au renforcement de la structure financière des PMI afin de limiter les comportements dictés par la recherche de la survie de la firme 
à court terme et de favoriser au contraire l'esprit entrepreneurial et donc la prise de risque ;

- inciter les PMI à accroître ou à préserver le degré de spécificité de leurs actifs, par exemple en stimulant leurs efforts en matière de dépenses en recherche et développement ou d'innovation.

\section{Annexe I \\ La base de données sur les stratégies d'entreprises}

La base de données sur les comportements stratégiques des PME est alimentée chaque année par voie d'enquêtes menées par un enquêteur de la Banque de France auprès de 2000 chefs d'entreprise. L'entretien, qui dure une heure trente, se déroule en tête-à-tête et avec un questionnaire assisté par ordinateur.

\subsection{Les thèmes du questionnaire}

Le questionnaire s'articule autour de neuf thèmes :

A - Démarche stratégique

(collecte de l'information par l'entrepreneur, processus de prise de décision, relations de groupe, répartition des fonctions)

$B$ - Fiche d'identité des activités (identification des activités, engagement à l'exportation)

C - Turbulence de l'environnement (turbulence technologique, sectorielle, macroéconomique...)

D - Analyse structurelle des relations entreprise - environnement (modèle des « cinq forces », M. Porter)

E - Stratégie concurrentielle par produits

(objectifs, manœuvres, facteurs clés de succès, source des avantages concurrentiels...)

$F$ - Synergies

(pour les entreprises pluriactivités)

G- Caractérisation du mode de production

$H$ - Structure et mode d'organisation

I- Croissance externe et alliances

Chaque thème est traité par des questions dont la structure est codifiée (recours à des échelles de Likert, à des questions de type binaire, à des listes à choix simple ou multiple ou, enfin, des listes de propositions à classer). Le questionnaire est fermé. Cela permet de transformer les réponses données par l'entreprise en 
valeurs numériques, alimentant une base de données, et aussi de construire des indicateurs élaborés et de mesurer des concepts stratégiques, à partir de la combinaison des valeurs prises par plusieurs questions. Néanmoins, l'enquêteur a la possibilité d'ajouter dans un bloc-notes des informations supplémentaires qui peuvent lui sembler importantes pour la connaissance individuelle de l'entreprise, comme pour l'amélioration des enquêtes futures.

\subsection{Les principales options méthodologiques}

Afin d'assurer une homogénéité des réponses et de limiter les biais inhérents à ce type d'enquête, les options suivantes ont été retenues :

- L'enquête n'est pas réalisée par courrier. Les données sont recueillies par entretien direct entre un enquêteur de la Banque de France, spécialement formé, et un dirigeant de l'entreprise. L'enquêteur est à même d'expliciter et de préciser la formulation des questions posées, afin d'assurer la fiabilité des réponses. L'interlocuteur choisi est le dirigeant de l'entreprise, afin de réduire le biais qui, pour certains thèmes, peut apparaître lorsque l'interlocuteur représente l'un ou l'autre service de l'entreprise (exemple : il est possible que les objectifs poursuivis par le service commercial diffèrent de ceux poursuivis par le service des finances, et c'est le dirigeant qui est le mieux à même d'exprimer l'objectif dominant). Bien entendu, le dirigeant a la possibilité de se faire assister par les collaborateurs de son choix et de fournir des réponses collégiales.

- Certaines questions ne sont pas posées au niveau global de l'entreprise, mais au niveau de son secteur et de ses domaines d'activité stratégique. En effet, dans le cas d'entreprises diversifiées, les variables stratégiques peuvent diverger selon le segment concerné. Dans ce cas, il est plus aisé pour le chef d'entreprise de donner une information élémentaire au niveau le plus fin, l'appréciation globale du comportement de la firme se faisant lors du traitement des données.

- Une contrainte a été introduite pour la détermination des domaines d'activité stratégique (notamment thème $\mathrm{E}$ du questionnaire): le découpage éventuel s'effectue en fonction de la nomenclature d'activité française (NAF), qui est la transposition en France de la NACE harmonisée en Europe. Ce choix, quelque peu réducteur et objet de débats, était indispensable pour envisager d'effectuer des comparaisons entre entreprises sur une base uniforme.

- Les questions reposant sur la perception du chef d'entreprise ont été limitées au maximum et complétées par des questions indirectes qui, combinées entre elles, sont de nature à limiter les biais. Dans la mesure du possible, 
les questions dites subjectives font appel à l'expertise du dirigeant sur son entreprise. Le postulat de départ est que le chef d'entreprise possède des informations uniques et irremplaçables sur sa société.

- La périodicité de l'enquête et la taille de l'échantillon ont été choisies en tenant compte de l'étendue du champ d'investigation retenu : l'échantillon est constitué de 4000 entreprises de l'industrie manufacturière, dont les effectifs sont compris entre 35 et 2000 salariés. Cette fourchette d'effectifs est assez large, afin de couvrir aussi bien les entreprises moyennes dans un contexte régional que les entreprises moyennes dans un contexte européen. $\mathrm{Au}$ sein de cette fourchette, les deux tiers des entreprises enquêtées comprennent entre 100 et 500 salariés. Les entreprises sont enquêtées une fois tous les deux ans, chaque année étant consacrée à la mise à jour d'une moitié de l'échantillon. Le taux de couverture, calculé par rapport au total des effectifs employés, est de l'ordre de $20 \%$ pour la plupart des secteurs représentés.

\section{Annexe II \\ Répartition sectorielle de l'échantillon}

\begin{tabular}{llcr}
\hline $\begin{array}{l}\text { Code } \\
\text { d'activité } \\
\text { INSEE }\end{array}$ & Secteur & $\begin{array}{c}\text { Nombre } \\
\text { d'entreprises }\end{array}$ & $\begin{array}{c}\text { Taux moyen } \\
\text { d'investissement }\end{array}$ \\
\hline 15 & Industrie agroalimentaire & 417 & $14,44 \%$ \\
17 & Industrie textile & 211 & $11,20 \%$ \\
18 & Industrie de l'habillement & 185 & $3,95 \%$ \\
19 & Industrie du cuir et de la chaussure & 68 & $6,06 \%$ \\
22 & Édition, imprimerie, reproduction & 194 & $8,82 \%$ \\
24 & Industrie chimique & 64 & $9,26 \%$ \\
29 & Fabrication de machines et équipements & 13 & $9,07 \%$ \\
32 & Fabrication d'équipements radio, télévision & & $24,47 \%$ \\
33 & $\quad$ et communication & 4 & $7,30 \%$ \\
& Fabrication d'instruments médicaux, & & $8,46 \%$ \\
34 & $\quad$ de précision, d'optique et d'horlogerie & 22 & $7,14 \%$ \\
36 & Industrie automobile & 89 & \\
\hline
\end{tabular}

Les secteurs de l'industrie agroalimentaire, du textile et de la fabrication d'équipements médicaux, de précision, d'optique et d'horlogerie - bien que, pour ce dernier, la faiblesse du nombre d'entreprises ne permette pas de considérer la moyenne comme représentative - semblent les plus dynamiques au regard de l'investissement, et c'est l'inverse pour le secteur de l'habillement. 


\section{Annexe III \\ Modèle de régression multiple: présentation des variables}

\subsection{Normalisation des variables quantitatives}

La normalisation a été effectuée par regroupement de centiles des distributions, en veillant toutefois à ce que la modalité centrale comprenne la valeur médiane de la variable d'origine.

N_TXAUTO (TXAUTOFI normalisée)

\begin{tabular}{ccccc}
\hline Value Label & Value & Frequency & $\begin{array}{c}\text { Valid } \\
\text { Percent }\end{array}$ & $\begin{array}{c}\text { Cum } \\
\text { Percent }\end{array}$ \\
\hline 1,00 & 28 & 1,9 & 2,0 & 2,0 \\
2,00 & 57 & 4,0 & 4,0 & 6,0 \\
3,00 & 114 & 7,9 & 8,0 & 14,0 \\
4,00 & 171 & 11,9 & 12,0 & 26,0 \\
5,00 & 214 & 14,9 & 15,0 & 41,0 \\
6,00 & 257 & 17,9 & 18,0 & 59,0 \\
7,00 & 214 & 14,9 & 15,0 & 74,0 \\
8,00 & 171 & 11,9 & 12,0 & 86,0 \\
9,00 & 114 & 7,9 & 8,0 & 94,0 \\
10,00 & 57 & 4,0 & 4,0 & 98,0 \\
11,00 & 28 & 1,9 & 2,0 & 100,0 \\
& 14 & 1,0 & Missing & \\
\hline Total & 1439 & 100,0 & 100,0 & \\
\hline
\end{tabular}

N_TXINV (TXINV normalisée)

\begin{tabular}{ccccc}
\hline Value Label & Value & Frequency & $\begin{array}{c}\text { Valid } \\
\text { Percent }\end{array}$ & $\begin{array}{c}\text { Cum } \\
\text { Percent }\end{array}$ \\
\hline 1,00 & 28 & 1,9 & 1,9 & 1,9 \\
2,00 & 44 & 3,1 & 3,1 & 5,0 \\
3,00 & 72 & 5,0 & 5,0 & 10,0 \\
4,00 & 131 & 9,1 & 9,1 & 19,1 \\
5,00 & 242 & 16,8 & 16,8 & 35,9 \\
6,00 & 406 & 28,2 & 28,2 & 64,1 \\
7,00 & 243 & 16,9 & 16,9 & 81,0 \\
8,00 & 131 & 9,1 & 9,1 & 90,1 \\
9,00 & 71 & 4,9 & 4,9 & 95,1 \\
10,00 & 43 & 3,0 & 3,0 & 98,1 \\
11,00 & 28 & 1,9 & 1,9 & 100,0 \\
\hline Total & 1439 & 100,0 & 100,0 & \\
\hline
\end{tabular}




\section{CORRELATIONS}

\begin{tabular}{lcc}
\hline & \multicolumn{2}{c}{- - Correlation Coefficients - - } \\
N_TXAUTO & N_TXAUTO & TXAUTOFI \\
& 1,0000 & 0,9499 \\
& $(1425)$ & $(1425)$ \\
N_TXINV & N_TXINV & P $=0,000$ \\
& 1,0000 & TXINV \\
& $(1439)$ & 0,8684 \\
& & $(1439)$ \\
\hline
\end{tabular}

\subsection{Binarisation des variables qualitatives issues de l'enquête}

\section{Information stratégique}

Trois classes ont été binarisées. Ces classes correspondent aux terciles de la distribution du score informationnel : classe faible (score inférieur ou égal à $2,8 / 5$ ), classe forte (score supérieur ou égal à $3,75 / 5$ ), classe intermédiaire (score compris entre $2,8$ et 3,75$)$.

\section{Croissance du marché principal}

Trois classes ont été binarisées : classe négative (taux de croissance moyen au cours des deux années précédentes inférieur à 0 ), classe positive (taux de croissance supérieur à 0 ), classe intermédiaire (taux de croissance nul). Ces classes représentent respectivement $37 \%, 40 \%$ et $23 \%$ de la distribution d'origine.

\section{Participation du dirigeant au capital}

Les classes binarisées correspondent à chacune des modalités de la variable d'origine: absence de participation (dirigeant mandaté), participation simple, influence notable, contrôle conjoint, contrôle exclusif.

\section{Effectifs}

Les classes binarisées correspondent aux classifications généralement retenues: effectifs inférieurs à 50 salariés, effectifs compris entre 50 et 99 salariés, effectifs compris entre 100 et 249 salariés et effectifs compris entre 250 et 499 salariés.

\section{Style de management}

Les classes binarisées correspondent aux quatre groupes d'entrepreneurs mis en évidence : attentistes, réactifs, opportunistes et proactifs. 


\section{Bibliographie}

ABEL, A. (1983), « Optimal investment under uncertainty », American Economic Review, vol. 73, p. 228-233.

ABEL, A. et J. EBerly (1994), «A unified model of investment under uncertainty », American Economic Review, vol. 84, p. 1369-1384.

AlCROFT, D. (1966), «Technical progress and British enterprise», Business History, vol. 8.

ARROw, K. (1968), « Optimal capital policy with irreversible investment », dans J.N. Wolfe (dir.), Value, Capital, and Growth, Papers in honour of Sir John Hicks, Edimbourg, Edinburgh University Press.

Belletante, B. (1991), «Pour une approche des spécificités financières de la PME au travers du concept de territoire financier », Revue internationale PME, vol. 4, $\mathrm{n}^{\circ} 1$, p. 30-49.

BELLETANTE, B. (1995), « Finance et PME : quels champs pour quels enjeux ? », Revue inter-

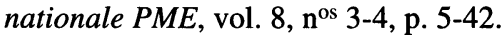

BERNANKE, B. (1983), «Irreversibility, uncertainty and cyclical investment », Quarterly Journal of Economics, p. 85-106.

Betbeze, J.P. (1990), L'investissement, Paris, Presses universitaires de France.

BouRdieU, J., B. COEURE et B. SEDILlot (1997), «Investissement, incertitude et irréversibilité », Revue économique, vol. $48, \mathrm{n}^{\circ} 1$, janvier, p. 23-53.

CABALlERO, R. (1991), «On the sign of the investment-uncertainty relationship », American Economic Review, vol. 81, p. 279-288.

CALOF, J. (1985), «On the positive role of financial intermediation in allocation of venture capital in market with imperfect information », Journal of Finance, vol. 38, $\mathrm{n}^{\circ} 5$, p. 1543-1568.

Chamley, C. et D. Gale (1994), «Information revelation and strategic delay in a model of investment », Econometrica, vol. 62, no 5, p. 1065-1086.

CHANDLER, A. (1976), « The development of modern management structure in the US and UK », dans L. Hannah, Management Strategy and Business Development, Londres, The Macmillan Press Ltd., 267 p.

DiETSCH, M. et E. KREMP (1997), «Le crédit interentreprises : les prêteurs et les bénéficiaires », Banque de France, Observatoire des Entreprises.

DIXIT, A. (1989), «Entry and exit decisions under uncertainty », Journal of Political Economy.

DIXIT, A. (1991), «Irreversible investment with price ceilings », Journal of Political Economy.

DIXIT, A. (1992), «Investment and hysteresis », Journal of Economic Perspectives, vol. 6, p. 107-132.

GABRIE, H. et J.L. JACQUIER (1994), La théorie moderne de l'entreprise, Paris, Economica.

GALIEGUE, X. (1996), «Irréversibilité de l'investissement et valeur d'option », Revue d'économie politique, vol. 106, $\mathrm{n}^{\circ} 5$, p. 844-863. 
GIRARD, B. (1997), « Pourquoi les PME restent-elles PME?», Annales des Mines, Gérer et comprendre, septembre.

HARTMAN, R. (1972), « The effect of price and cost uncertainty on investment », Journal of Economic Theory, vol. 5, p. 258-266.

HENRY, C. (1974), «Investment decisions under uncertainty : the irreversibility effect », American Economic Review, vol. 64, p.1006-1012.

HOLMES, S. et P. KENT (1991), «An empirical analysis of the financial structure of small and large Australian manufacturing enterprises », Journal of Small Business Finance, vol. $1, \mathrm{n}^{\circ} 2$, p. 141-154.

JENSEN, M. et W. MECKLING (1976), «Agency costs and the theory of the firm », Journal of Financial Economics.

JORGENSEN, D. (1963), « Capital theory and investment behaviour », American Economic Review, vol. 53, p. 247-259.

Julien, P.A. et M. MARCHESNAY (1987), La petite entreprise, Paris, Vuibert.

LANDES, D. (1957), «Observations on France : economics, society and policy», World Politics, avril.

MAC DONALD, R. et D. SIEGEL (1985), « Investment and the valuation of firms when there is an option to shut down ", International Economic Review, vol. 26, juin, p. 331-349.

MAC DONALD, R. et D. SIEGEL (1986), «The value of waiting to invest », Quarterly Journal of Economics, vol. 101, n ${ }^{\circ} 4$, p. 707-728.

MALINVAUD, E. (1987), « Capital productif, incertitude et profitabilité », Annales d'économie et de statistique, vol. 5, p. 1-36.

MILLS, E.S. (1959), «Uncertainty and price theory », Quarterly Journal of Economics, $\mathrm{n}^{\circ}$ 73, p. 116-130.

MILLS, E.S. (1962), Price, Output and Inventory Policy, New York, John Wiley and Sons.

MYERS, S.C. et N.S. MAJLUF (1984), « Corporate financing and investment decision when firms have informations that investors do not have », Journal of Financial Economics, vol. 13, p. 187-221.

PINDYCK, R. (1988), «Irreversible investment, capacity choice and the value of the firm », American Economic Review, p. 969-985.

PINDYCK, R. (1991), «Irreversibility, uncertainty and investment », Journal of Economic Literature, vol. 29, p. 1110-1148.

RICHET, X. (1994), Économie de l'entreprise, Paris, Hachette, p. 125.

SILVESTRE, H. et R. GOUJET (1996), « Lisibilité de l'environnement, management stratégique : éléments de recherche sur les PMI », Revue internationale PME, vol. 9, $\mathrm{n}^{\circ} 1, \mathrm{p} .61-78$.

Tenenhaus, M. (1994), Méthodes statistiques en gestion, Paris, Dunod.

TOBIN, J. (1969), « A general equilibrium approach to monetary theory », Journal of Money, Credit and Banking, vol. 1, p. 15-29. 